\title{
Adaptive Continuous Higher Order Sliding Mode Control
}

\author{
C. Edwards*, Y. Shtessel \\ Structures \& Dynamics Research Group, CEMPS, University of Exeter, Exeter, UK \\ Department of Electrical Engineering, University of Alabama, Huntsville, AL, USA
}

\begin{abstract}
This paper is concerned with the development of an adaptation structure which can be applied to conventional, super-twisting and higher-order sliding mode schemes. The objective is to alter the modulation gains associated with these schemes in such a way that they are as small as possible to mitigate chattering effects, but large enough to ensure that sliding can be maintained in the presence of bounded and derivative bounded uncertainties. In all the proposed schemes, the equivalent control is used to drive the adaptive mechanism. The approach is based on a novel dual layer nested adaptive methodology which is quite different to the existing schemes proposed in the sliding mode literature. The new adaptive schemes do not require knowledge of the minimum and maximum allowed values of the adaptive gain, and in their most general form, do not require information about the bound on the disturbances and their derivatives.
\end{abstract}

Keywords: Sliding modes, Higher order sliding modes, adaptive control

\section{Introduction}

The insensitivity and finite time convergence properties enjoyed by sliding mode controllers make them a useful approach for systems with significant uncertainties. However these robustness properties come at a cost, usually termed 'chattering', resulting from high frequency switching of the control signal. Higher order sliding mode control techniques (Shtessel et al. (2013)) offer significant chattering mitigation through artificially increasing the input-output relative degree, and are capable of providing control signals which are continuous. Families of higher order sliding mode controllers with specific 'templates' involving either recursive or nested structures have been proposed (Levant (2003, 2006)). However, these control laws all contain signum structures pre-multiplied by some bound on the uncertainties (or bounds on the derivatives of the uncertainty) and so chattering is not totally eliminated even by increasing the relative degree. Usually during the design process, conservative upper bounds on these quantities are used to guarantee that sliding will take place, but this conservatism exacerbates the chattering associated with the implementation. This trade-off has motivated research in what could be described as 'adaptive sliding mode control' whereby the gains in the controller, representing bounds on the uncertainty, attempt to adapt to a level where they are as small as possible and yet guarantee sliding is maintained. This research area has a long research history and key papers in this area are (Huang et al. (2008); Plestan et al. (2010)). A recent overview of this field is given

\footnotetext{
*Corresponding author: Tel.: +44 1392726632.

Email addresses: c.edwards @exeter.ac.uk (C. Edwards*), shtessel@eng . uah. edu (Y. Shtessel)

${ }^{1}$ An early version of this paper was presented at ACC'14.
}

in Utkin \& Poznyak (2013a). The creation of new Lyapunov functions for higher order sliding mode control structures - particularly twisting and super-twisting controllers - has also rejuvenated interest in this area (Moreno \& Osorio (2008)) and the literature expanding these Lyapunov ideas into the realm of adaptive sliding mode control is developing rapidly (Plestan et al. (2010); Alwi \& Edwards (2013); Shtessel et al. (2012); Bartolini et al. (2103); Taleb et al. (2013)). Whilst it is intuitively clear that when sliding begins to deteriorate the controller gains must be increased, devising an effective way of lowering unnecessarily large gains once sliding is achieved, has proved more elusive. This paper follows the approach of Utkin \& Poznyak (2013a) and Lee \& Utkin (2007) in the sense that the adaption scheme relies on the availability, in real time, of the equivalent control signal, and exploits this information in the adaptive scheme.

The main contribution of this paper is to propose new equivalent control-based adaptive schemes for conventional, supertwisting and continuous higher order sliding mode control algorithms. The approach is based on a novel dual layer nested adaptive scheme which is quite different to the existing schemes proposed in the sliding mode literature (Plestan et al. (2010); Utkin \& Poznyak (2013a)). The scheme allows the magnitude and rate of change of the controller parameters to adapt whilst guaranteeing a sliding motion. The new adaptive schemes do not require knowledge of the minimum and maximum allowed values of the adaptive gain and in their most general form, do not require information about the bound on the disturbances and their derivatives that are necessary in Utkin \& Poznyak (2013a). The notation used in the paper is standard - the symbol $\mathbb{R}$ is used to represent the real numbers and $\|\cdot\|$ is used to represent the Euclidean norm. 


\section{A Single-Input Single-Output Formulation}

Consider initially the prototypical first-order sliding mode equation representing the dynamics of the switching variable

$$
\dot{\sigma}(t)=a(t)+u(t)
$$

where $\sigma(t) \in \mathbb{R}$ represents the switching function to be forced to zero in finite time, $u(t)$ represents the scalar control input to be manipulated and $a(t)$ is a disturbance ${ }^{2}$. Here it will be assumed that $a(t)$ is not known but its magnitude and its derivative are bounded so that $|a(t)|<a_{0}$ and $|\dot{a}(t)|<a_{1}$ where $a_{0}$ and $a_{1}$ are finite.

Consider the control law

$$
u(t)=-(k(t)+\eta) \operatorname{sign}(\sigma(t))
$$

where $\eta$ is a small positive design constant and $k(t)$ is a varying scalar term which satisfies an adaptive scheme, which will be explicitly defined later in the section. The objective is to select $k(t)$ as small as possible to ensure sliding takes place in (1). Note that a sufficient condition to enforce a sliding motion in (1) is that

$$
k(t)>|a(t)|
$$

in which case the $\eta$-reachability condition $\sigma \dot{\sigma}<-\eta|\sigma|$ is satisfied (Edwards \& Spurgeon (1998)). During the sliding motion $\sigma(t) \equiv 0$ and the so-called equivalent control $u_{e q}(t)$, which is the value the switched signal in (2) must take on average (Utkin (1992)) to maintain sliding, and which is formally obtained as the solution to the algebraic equation $\dot{\sigma}=0$ when $\sigma=0$, must satisfy

$$
u_{e q}(t)=-a(t)
$$

i.e it must exactly cancel the unknown disturbance/uncertainty. Consequently during sliding $\left|u_{e q}(t)\right|=|a(t)|$. Although the equivalent control was conceived as an abstraction to allow the analysis of the reduced order sliding motion, a close approximation can be obtained in real-time by low-pass filtering of the switching signal $u(t)$ (Utkin (1992)). In this way if $\bar{u}_{e q}(t)$ satisfies

$$
\dot{\bar{u}}_{e q}(t)=\frac{1}{\tau}\left(-(k(t)+\eta) \operatorname{sign}(\sigma(t))-\bar{u}_{e q}(t)\right)
$$

where $\tau>0$ is a (small) time constant, $\left|\bar{u}_{e q}(t)-u_{e q}(t)\right|$ can be small for small enough $\tau$ (Utkin (1992)). The filter in (4) is the simplest choice, but other higher order systems with lowpass characteristics can be employed. In this paper it will be assumed there exist scalars $1>\epsilon_{1}>0$ and $\epsilon_{0}>0$ such that

$$
\left\|\bar{u}_{e q}(t)|-| u_{e q}(t)\right\|<\epsilon_{1}\left|u_{e q}(t)\right|+\epsilon_{0}
$$

holds for all time after a finite time $t_{e q}$, to allow for the (fast) dissipation of the effects of the initial conditions of the filter. Equation (5) is an assumption on the accuracy to which $u_{e q}(t)$

${ }^{2}$ Equation (1) does not represent the dynamics of the overall system to be controlled, but instead represents the dynamics of the sliding variable - possibly after partial closed-loop compensation with control elements representing the known parts of the equivalent control (Edwards \& Spurgeon (1998)). is estimated and depends on the filter bandwidth $\frac{1}{\tau}$. For a given $t_{e q}$ and $\tau$, estimates of $\epsilon_{0}$ and $\epsilon_{1}$ can easily be obtained to ensure (5) holds. Furthermore in an engineering system information about the bandwidth of the uncertainty can be exploited to help select $\tau$ an ensure an appropriate level of accuracy. Here the equivalent control will be used to construct the adaptive algorithm for $k(t)$. To introduce a 'safety margin', the objective will be to ensure

$$
k(t)>\frac{1}{\alpha}\left|\bar{u}_{e q}(t)\right|+\epsilon
$$

where $0<\alpha<1$ and $\epsilon>0$ are design scalars (depending on $\epsilon_{0}$ and $\left.\epsilon_{1}\right)$ chosen to ensure the estimate $\bar{u}_{e q}(t)$ satisfies

$$
\frac{1}{\alpha}\left|\bar{u}_{e q}(t)\right|+\epsilon / 2>\left|u_{e q}(t)\right|
$$

Now define an error variable

$$
\delta(t)=k(t)-\frac{1}{\alpha}\left|\bar{u}_{e q}(t)\right|-\epsilon
$$

Note if $\delta=0$ then $k(t)=\frac{1}{\alpha}\left|\bar{u}_{e q}(t)\right|+\epsilon>\left|u_{e q}(t)\right|=|a(t)|$. Thus, in what follows, the problem of maintaining sliding is transposed to one of forcing $\delta(t) \rightarrow 0$, or at least sufficiently close to zero, so that $k(t)+\eta>|a(t)|$ can be guaranteed.

The adaptive control element $k(t)$ in (2) will now be explicitly described. Specifically define

$$
\dot{k}(t)=-\rho(t) \operatorname{sign}(\delta(t))
$$

where $\rho(t)$ is a varying scalar. This scalar has a physical interpretation: namely it represents an upper-bound on the rate of change of the disturbance. In this paper it is assumed that $\rho(t)$ has the structure

$$
\rho(t)=r_{0}+r(t)
$$

where $r_{0}$ is a fixed positive scalar. The evolution of $r(t)$ will also satisfy a differential equation (i.e an adaptive law) that will be described shortly. Precise details regarding the adaptation law for $r(t)$ proposed in this paper will depend on the assumptions which are made with respect to the knowledge about $a_{1}$ (the upper-bound $|\dot{a}(t)|)$. In particular two layers of adaptation occur. One layer is concerned with the magnitude of the switching signal in the control law $k(t)$. The rate at which $k(t)$ can change depends on the time-varying parameter $r(t)$ which itself adapts in a way to ensure $r(t)+r_{0}>|\dot{a}(t)|$. This is the second adaptive layer in the scheme. This second layer obviates the need to know a-priori the bound $a_{1}$. This dual layer adaptation is the crux of this paper. Two situations will be considered: firstly the situation when $a_{1}$ is known, and secondly when it is unknown.

\subsection{Formulation when the bound $a_{1}$ is known}

In this subsection it is assumed that $a_{0}$ is unknown (but bounded), but $a_{1}$ is available: i.e the worst case rate of change of the disturbance $a(t)$ is known. Define

$$
e(t)=q a_{1} / \alpha-r(t)
$$

where the user defined scalar $q>1$ is a safety margin chosen to ensure $\left|\frac{d}{d t}\left(\bar{u}_{e q}(t)\right)\right|<q a_{1}$. Since (in this subsection) it is assumed 
that $a_{1}$ is known, $e(t)$ is known, and can be exploited in the adaptation scheme. Define

$$
\dot{r}(t)=\gamma|\delta(t)|+r_{0} \sqrt{\gamma} \operatorname{sign}(e(t))
$$

where $\gamma>0$ is a design scalar and $\delta(t)$ is defined in (8).

Proposition 1. Consider the sliding mode equation (1) subject to an uncertainty $a(t)$ which satisfies $|a(t)|<a_{0}$ and $|\dot{a}(t)|<$ $a_{1}$, where $a_{0}$ and $a_{1}$ are finite, and $a_{1}$ is known. Then the control law (2), exploiting the dual layer adaptive scheme given by (9) and (12), forces $\delta(t)=0$ in finite time, and consequently ensures a sliding motion can be sustained. Furthermore the gains $\rho(t)$ and $k(t)$ in (9) and (10) remain bounded.

Proof: The dynamical system associated with the variables $\delta(t)$ and $e(t)$ in (8) and (11) are governed by the differential equations

$$
\begin{aligned}
& \dot{e}(t)=-\gamma|\delta(t)|-r_{0} \sqrt{\gamma} \operatorname{sign}(e(t)) \\
& \dot{\delta}(t)=-\left(r_{0}+\frac{q a_{1}}{\alpha}-e(t)\right) \operatorname{sign}(\delta(t))-\frac{1}{\alpha} \phi(t)
\end{aligned}
$$

where $\phi(t):=\frac{d}{d t}\left(\bar{u}_{e q}(t)\right)$ and satisfies $|\phi(t)|<q a_{1}$. These equations have discontinuous right hand sides and so the solution needs to be understood in a Filippov sense (Filippov (1964)). The stability of (13)-(14) will now be analyzed using

$$
V=\frac{1}{2} \delta^{2}+\frac{1}{2 \gamma} e^{2}
$$

It follows from (14) that

$$
\begin{aligned}
\delta \dot{\delta} & \leq-r_{0}|\delta(t)|+\left(e(t)-\frac{q a_{1}}{\alpha}\right)|\delta(t)|+|\delta(t)| \frac{q a_{1}}{\alpha} \\
& =-r_{0}|\delta(t)|+e(t)|\delta(t)|
\end{aligned}
$$

from the definition of $e(t)$ in (11). Therefore from (12)

$$
\begin{aligned}
\dot{V} & \leq-r_{0}|\delta(t)|+|\delta(t)| e(t)-\frac{1}{\gamma} e(t) \dot{r}(t) \\
& =-\sqrt{2} r_{0}\left(\frac{1}{\sqrt{2}}|\delta(t)|+\frac{1}{\sqrt{2 \gamma}} \mid(e(t) \mid) \leq-r_{0} \sqrt{2 V}\right.
\end{aligned}
$$

since

$$
-\left(\frac{|\delta|}{\sqrt{2}}+\frac{|e|}{\sqrt{2 \gamma}}\right) \leq-\left(\frac{|\delta|^{2}}{2}+\frac{|e|^{2}}{2 \gamma}\right)^{1 / 2} \quad \text { for all } \delta, e
$$

Inequality (17) implies $V=0$ in finite time and therefore $\delta(t)$ and $e(t)$ become zero in finite time. Consequently from the definition of $\delta$ in (8)

$$
k(t)=\frac{1}{\alpha}\left|\bar{u}_{e q}(t)\right|+\epsilon>\left|u_{e q}(t)\right|=|a(t)|
$$

This is exactly the condition necessary to maintain sliding. Also note that since $e(t)$ is bounded, from the definition of $e(t)$ in (11), $r(t)$ and hence $\rho(t)$ is bounded.

\subsection{Formulation when $a_{1}$ is unknown}

In this subsection it is assumed that both $a_{0}$ and $a_{1}$ are unknown (but bounded). Since $a_{1}$ is not known the variable $e(t)$ is unknown and the adaptive scheme in (12) can not be used. Instead define

$$
\dot{r}(t)= \begin{cases}\gamma|\delta(t)| & \text { if }|\delta(t)|>\delta_{0} \\ 0 & \text { otherwise }\end{cases}
$$

where $\delta_{0}>0$ is a design scalar. The adaptive scheme now comprises (9), (10) and (20).

Proposition 2. Consider the system in equation (1) subject to an uncertainty $a(t)$ which satisfies $|a(t)|<a_{0}$ and $|\dot{a}(t)|<a_{1}$, where $a_{0}$ and $a_{1}$ are finite, but both $a_{0}$ and $a_{1}$ are unknown. If $\epsilon$ is chosen to satisfy

$$
\frac{1}{4} \epsilon^{2}>\delta_{0}^{2}+\frac{1}{\gamma}\left(\frac{q a_{1}}{\alpha}\right)^{2}
$$

for any given $\delta_{0}$ and $a_{1}$, then the control law (2), exploiting the dual layer adaptive scheme given by (9) and (20), forces $|\delta(t)|<\epsilon / 2$ in finite time and consequently ensures a sliding motion can be sustained. Furthermore the gains $\rho(t)$ and $k(t)$ remain bounded.

Proof: The Lyapunov function (15) will be employed to analyze the error variables $e(t)$ and $\delta(t)$ from (8) and (11). As before, from (16), it follows that

$$
\delta \dot{\delta} \leq-r_{0}|\delta(t)|+e(t)|\delta(t)|
$$

Suppose $r(0)=0$, then whatever the evolution of $(\delta(t), e(t))$, it follows from the definition of $e(t)$ in (11) that $e(t) \leq \frac{q a_{1}}{\alpha}$ because from (20) $r(t) \geq 0$ for all time.

If $|\delta(t)|>\delta_{0}$ then from the definition of $e(t)$ in (11) and of $r(t)$ from (20), it follows that $\dot{e}(t)=-\dot{r}(t)=-\gamma|\delta(t)|$ and

$$
\begin{aligned}
\dot{V} & \leq-r_{0}|\delta(t)|+|\delta(t)| e(t)-\frac{1}{\gamma} e \dot{r}(t) \\
& =-r_{0}|\delta(t)|+|\delta(t)| e(t)-|\delta(t)| e(t)=-r_{0}|\delta(t)|
\end{aligned}
$$

Otherwise inside the vertical strip of width $2 \delta_{0}$ in Fig. 1, when $|\delta(t)|<\delta_{0}$, it follows from (22) that if $e(t)<0$, then, as before, $\dot{V} \leq-r_{0}|\delta(t)|$.

Since $e(t) \leq \frac{q a_{1}}{\alpha}$ for all time (because $\dot{r} \geq 0$ ), in the solution domain, outside of the rectangle

$$
\mathcal{R}=\left\{(\delta, e):|\delta|<\delta_{0}, 0 \leq e<\frac{q a_{1}}{\alpha}\right\}
$$

it follows that $\dot{V} \leq-r_{0}|\delta(t)|$. Let $\bar{V}$ be the smallest ellipse centred at the origin of the form

$$
\overline{\mathcal{V}}=\{(\delta, e): V(\delta, e)<\bar{r}\}, \bar{r}>0
$$

which encloses the rectangle $\mathcal{R}$. In fact the "radius" $\bar{r}$ can be given explicitly as

$$
\bar{r}:=\frac{1}{2} \delta_{0}^{2}+\frac{1}{2 \gamma}\left(\frac{q a_{1}}{\alpha}\right)^{2}
$$

By construction $\mathcal{R} \subset \overline{\mathcal{V}}$, and so outside of $\overline{\mathcal{V}}$ in the solution domain, $\dot{V} \leq 0$, and therefore $\overline{\mathcal{V}}$ is an invariant set. Let $\epsilon$ be chosen so that (21) is satisfied.

If the solution $(\delta(t), e(t))$ enters $\overline{\mathcal{V}}$ (in finite time) then since $\overline{\mathcal{V}}$ is an invariant set, $(\delta(t), e(t))$ cannot leave $\overline{\mathcal{V}}$ and therefore from (21)), $|\delta(t)|<\epsilon / 2$. 


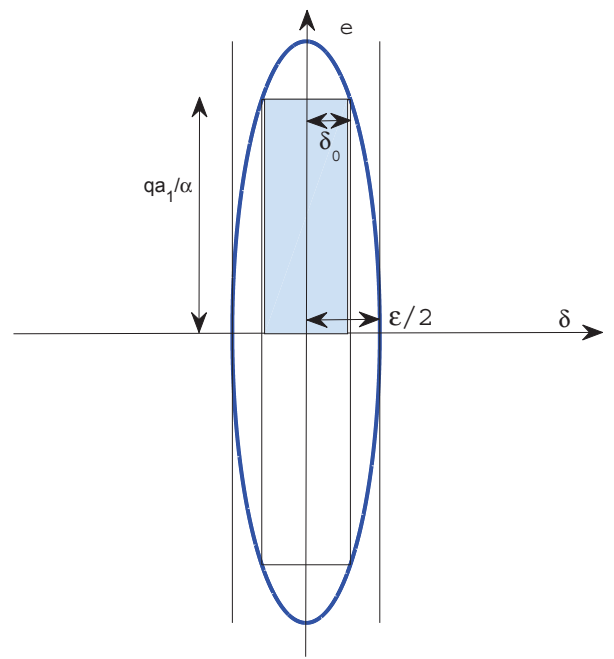

Figure 1: Visualization of $\overline{\mathcal{V}}$ and $\mathcal{R}$

Otherwise if the solution $(\delta(t), e(t))$ does not enter $\overline{\mathcal{V}}$, then from the arguments above $\dot{V} \leq-r_{0}|\delta(t)|$ and

$$
\int_{0}^{\infty} r_{0}|\delta(t)| d t \leq V(0)
$$

Since $V(e, \delta)$ is bounded for all time, the solution $(e(t), \delta(t))$ to (13)-(14) is bounded and consequently the right hand sides of (13)-(14) are bounded, which implies the derivatives $\dot{\delta}(t)$ and $\dot{e}(t)$ of the solution $(e(t), \delta(t))$ are bounded, and therefore $\delta(t)$ and hence $|\delta(t)|$ is uniformly continuous. Consequently (25) implies $\delta(t) \rightarrow 0$ as $t \rightarrow \infty$ (Barbalat's Lemma (Khalil (1992))). Consequently there exists a finite time $t_{0}$ such that $|\delta(t)| \leq \epsilon / 2$ for all time $t>t_{0}$. Therefore, irrespective of whether $(e(t), \delta(t))$ enters $\overline{\mathcal{V}}$ or not, $|\delta(t)|<\epsilon / 2$ in finite time.

Consequently from the definition of $\delta(t)$ in (8)

$$
|\delta(t)|=\left|k(t)-\frac{1}{\alpha}\right| \bar{u}_{e q}(t)|-\epsilon|<\epsilon / 2
$$

Thus

$$
k(t)-\frac{1}{\alpha}\left|\bar{u}_{e q}(t)\right|-\epsilon>-\epsilon / 2
$$

and therefore from (7)

$$
k(t)>\frac{1}{\alpha}\left|\bar{u}_{e q}(t)\right|+\frac{\epsilon}{2}>\left|u_{e q}(t)\right|=|a(t)|
$$

From (26) it follows that the condition for maintaining a sliding motion on $\sigma=0$ is guaranteed. Note since both $e(t)$ and $\delta(t)$ remain bounded, from (11), the adaptive gain $r(t)$ satisfies

$$
|r(t)|<\frac{a_{1}}{\alpha}+|e(t)|
$$

and so $r(t)$ and hence $\rho(t)$ remains bounded. Also from the definition of $\delta(t)$ in (8) it follows from (5)

$$
|k(t)|<|\delta(t)|+\frac{1}{\alpha}\left|\bar{u}_{e q}(t)\right|+\epsilon<|\delta(t)|+\left(1+\epsilon_{1}\right) \frac{a_{0}}{\alpha}+\epsilon
$$

and so $k(t)$ remains bounded.
Remark 1: Inequality (21) depends on different variables with different interpretations. The scalar $\delta_{0}$ needs to be larger than noise or computational errors. The user defined parameters $\epsilon, \alpha$ are the safety factors whilst $q$ reflects the accuracy associated with the estimation of the equivalent control. In particular $a_{1}$ is unknown. However by selecting the adaptive gain $\gamma$ sufficiently large (to dominate $a_{1}$ ), for any value of $\delta_{0}, q$ and $a_{1}$ there always exists an $\epsilon$ to ensure (21) is satisfied. Of course (21) is only a sufficient condition, and to formally ensure that inequality (21) is satisfied, the order of magnitude of $a_{1}$ must be known to allow $\gamma$ to be chosen sufficiently large. Alternatively the values of $\gamma$ and $\epsilon$ can be tuned via simulation because the physical effect of changing these values is easily understood.

Remark 2: In equality (26), the right hand side establishes a bounding cone around the equivalent control $\left|u_{e q}(t)\right|$ involving both multiplicative $\frac{(1-\alpha)}{\alpha}$ and fixed $\frac{\epsilon}{2}$ components. This introduces robustness into the adaptive scheme since the value of $\left|u_{e q}(t)\right|$ can only be estimated by $\bar{u}_{e q}(t)$ through the low-pass filtering process in (4). The amount of allowable uncertainty is a function of the parameters $\alpha$ and $\epsilon$ which are to be selected by the designer (subject to $0<\alpha<1$ and $\epsilon>0$ ). In this way the designer can introduce his/her own preferred level of "safety" into the algorithm by widening the cone through making $\alpha$ smaller and $\epsilon$ bigger - although, of course, this must be traded-off against how much larger $k(t)$ becomes with respect to $|a(t)|$.

Remark 3: Note that prior to sliding taking place, the adaptive scheme for $k(t)$ as described above is also applicable. This is similar to the result in Utkin \& Poznyak (2013a). Suppose sliding is not taking place in the interval $0 \leq t \leq t_{0}$. During this time $|\operatorname{sign}(\sigma(t))|=1$ almost everywhere and therefore from (4), $\left|\bar{u}_{e q}(t)\right|=k(t)+\eta$ almost everywhere ${ }^{3}$. Substituting this value in (8), it follows that almost everywhere

$$
\delta(t)=\frac{(\alpha-1)}{\alpha}(k(t)+\eta)-\epsilon<0
$$

since $0<\alpha<1$. Therefore $\operatorname{sign}(\delta(t))=-1$ and from (9)

$$
\dot{k}(t)=\rho(t)>r_{0}
$$

for $0 \leq t \leq t_{0}$ and therefore the gain $k(t)$ grows at a rate dictated by $\rho(t)$ which is always greater than $r_{0}$. Increasing $k(t)$ is precisely what is needed to induce sliding.

Remark 4: Note that whilst certain parallels exist between the adaptive schemes in (9), (12) and (20) and those proposed in Utkin \& Poznyak (2013a), the precise details are quite different. In this paper, compared to Utkin \& Poznyak (2013a), no knowledge about the upper-bound $a_{0}$ is required. Furthermore in the scheme presented in Section 2.2, no knowledge of the upper-bound on $a_{1}$ is required in the adaptive scheme. In Utkin \& Poznyak (2013a) knowledge of both $a_{0}$ and $a_{1}$ are required (as is the polarity of the disturbance signal - which is not allowed to change). Also the dual-layer nested adaptive architecture involving $k(t)$ and $r(t)$ is clearly quite different from the schemes proposed in Plestan et al. (2010).

${ }^{3}$ Of course prior to sliding, $\bar{u}_{e q}(t)$ in (4) no longer estimates the equivalent control $u_{e q}(t)$, which has no formal meaning during the reaching phase. 


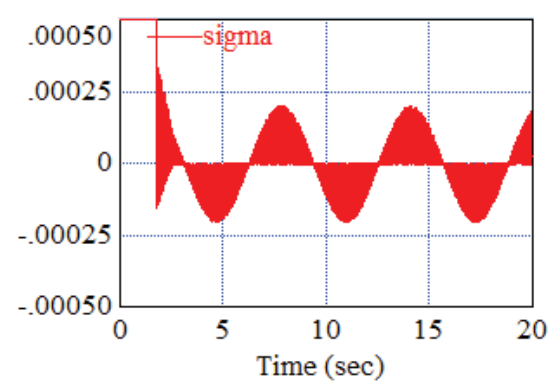

Figure 2: Evolutions of the switching function

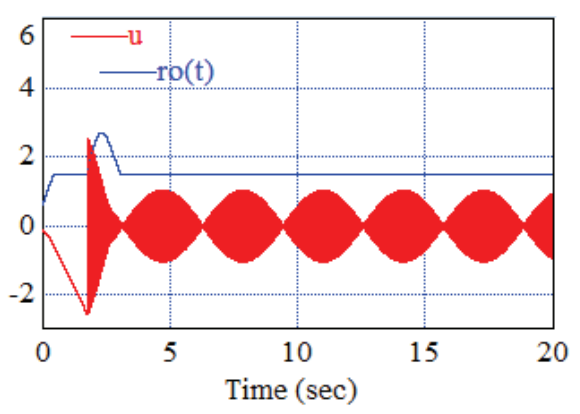

Figure 3: Evolutions of $u(t)$ and $\rho(t)$

\subsection{An Example}

Consider the sliding variable dynamics in (1) when the disturbance is given by $a(t)=\sin (t)$. Suppose initially that the bound $a_{1}=1$ on the rate of change of the disturbance $a(t)$ is known, so that the adaptive scheme given by (9) and (12) can be employed. In the simulations which follow $a_{1}=1, \alpha=0.99$, $r_{0}=0.5, \delta_{0}=0.01, \epsilon=0.15, \eta=0.01$ and $\gamma=200$. The time constant of the filter is $\tau=0.01$, starting with zero initial conditions. Figures 2-4 show the evolution of $\sigma(t)$, the control signal, and the two adaptive gains $k(t)$ and $\rho(t)$. Note that in this case $\rho(t)$ tracks the upper-bound $a_{1}=1$. Figure 4 shows $k(t)$ closely follows $|a(t)|$.

Now suppose that $a_{1}$ exists but is unknown, and the adaptive control scheme in (9) and (20) is employed. Note that in this case $\rho(t)$ no longer tracks the upper-bound $a_{1}=1$ (which is in accordance with the theory). However Figure 6 shows $k(t)$ still

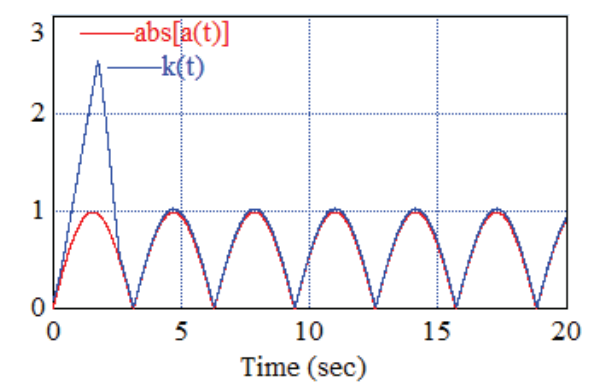

Figure 4: Evolutions of $k(t)$ and $|a(t)|$

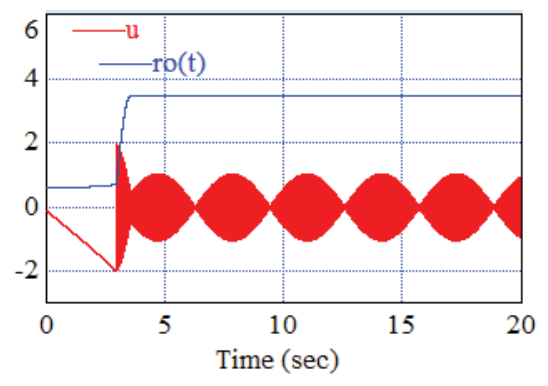

Figure 5: Evolutions of $u(t)$ and $\rho(t)$

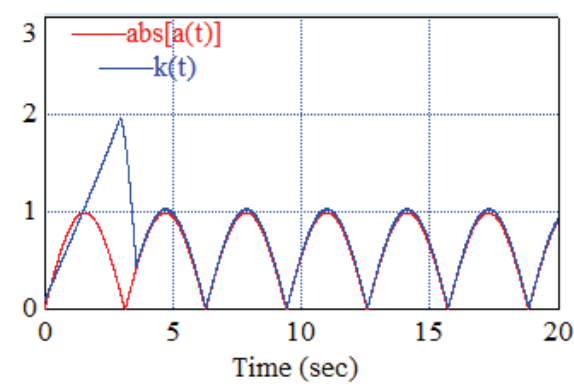

Figure 6: Evolutions of $k(t)$ and $|a(t)|$

closely follows $|a(t)|$.

\section{A Multi-Input Multi-Output Formulation}

Now consider a multi-variable version of the first-order scalar equation in (1) given by

$$
\dot{\sigma}(t)=a(t)+u(t)
$$

where $\sigma(t) \in \mathbb{R}^{m}$ represents the switching function to be forced to zero in finite time, $u(t) \in \mathbb{R}^{m}$ represents the control input to be manipulated and $a(t) \in \mathbb{R}^{m}$ is a disturbance which is imprecisely known. Here it will be assumed that $a(t)$ is not known but its magnitude and its derivative $\dot{a}(t)$ are bounded so that $\|a(t)\|<a_{0}$ and $\|\dot{a}(t)\|<a_{1}$ where $a_{0}$ and $a_{1}$ are finite but unknown.

Consider the unit vector control law (Edwards \& Spurgeon (1998)) given by

$$
u(t)=-(k(t)+\eta) \frac{\sigma(t)}{\|(\sigma(t))\|}
$$

where $\eta>0$ is a design constant and $k(t)$ is a varying term which satisfies an adaptive law. A sufficient condition to ensure sliding on $\sigma \equiv 0$ in finite time is

$$
k(t)>\|a(t)\|
$$

If (29) is satisfied then the multivariable $\eta$-reachability condition (Edwards \& Spurgeon (1998)) $\sigma^{T} \dot{\sigma} \leq-\eta\|\sigma\|$ is satisfied and sliding is guaranteed. In this multivariable situation the 
concept of equivalent control is equally applicable and once sliding is achieved $\left\|u_{e q}(t)\right\|=\|a(t)\|$. Now define an error signal

$$
\delta(t)=k(t)-\frac{1}{\alpha}\left\|\bar{u}_{e q}(t)\right\|-\epsilon
$$

where, as in Section 2, the scalars $0<\alpha<1$ and $\epsilon>0$ and $\bar{u}_{e q}(t)$ represents a low-pass filtered version of $u(t)$ in (28). As in Section 2.2 the gain $k(t)$ adapts according to the law in (9) where $\rho(t)$ adapts according to (20). Although the control problem is a multivariable one, the approach in Section 2 can be employed here because $\delta(t)$ defined in (30) is again a scalar, and a multivariable version of Proposition 2 can be proved.

\section{Adaptive Super-Twisting}

Consider once again the scalar sliding variable dynamics in (1) under the assumption that $|\dot{a}| \leq a_{1}$ where $a_{1}>0$ and $|\ddot{a}|<a_{2}$. Now suppose that in instead of the relay structure in (2), $u(t)$ is given by (the slightly modified) super-twisting control law

$$
\begin{aligned}
& u(t)=-\lambda|\sigma(t)|^{1 / 2} \operatorname{sign}(\sigma(t))+z(t) \\
& \dot{z}(t)=-k(t) \operatorname{sign}(\sigma(t))
\end{aligned}
$$

Here, as in Utkin \& Poznyak (2013a), the modulation gain associated with the discontinuous term is time-varying. As argued in Utkin \& Poznyak (2013a), for any given $\sigma(0)$, there exists a sufficiently large $\lambda>0$, such that, provided

$$
k(t)>a_{1}>|\dot{a}(t)|,
$$

the control structure in (31)-(32) drives both $\sigma$ and $\dot{\sigma}$ to zero in finite time: i.e. it induces a 2-SM.

Remark 5: Of course if $a_{1}$ is known, a standard fixed gain super-twisting approach could be employed. Although it can be argued, even in this situation there is benefit to using the "smallest" magnitude of switching gain which can sustain sliding rather than a possibly conservative upper bound on the worst case disturbance (Alwi \& Edwards (2013)).

Equations (1), (31) and (32) can be written in the form

$$
\begin{aligned}
\dot{\sigma}(t) & =-\lambda|\sigma(t)|^{1 / 2} \operatorname{sign}(\sigma(t))+v(t) \\
\dot{v}(t) & =\varphi(t)-w(t)
\end{aligned}
$$

where $\varphi(t)=\dot{a}(t)$, and

$$
w(t)=k(t) \operatorname{sign}(\sigma(t))
$$

During a 2-SM $\sigma=v=0$ (which is identical to the condition $\sigma=\dot{\sigma}=0$ ) and therefore the equivalent control $w_{e q}(t)=\varphi(t)$. Again two cases will be considered: firstly the case when $a_{2}$ (the bound on $|\ddot{a}|$ ) is known; and secondly the case when $a_{2}$ is unknown.

\subsection{Formulation when $a_{2}$ is known}

As in the earlier sections, it is assumed $\bar{w}_{e q}(t)$ is available by low-pass filtering $w(t)$ and satisfies similar bounds with respect to $w_{e q}(t)$ as in (7). In this section define

$$
\delta(t)=k(t)-\frac{1}{\alpha}\left|\bar{w}_{e q}(t)\right|-\epsilon
$$

where $0<\alpha<1$ and $\epsilon>0$ is a small real number. The first layer of the double-layer control gain adaptation algorithm is defined in (9), (10) and (12) where

$$
e(t)=\bar{q} a_{2} / \alpha-r(t)
$$

where as before $\bar{q}>1$ represents a user defined gain. Note that in order to realize (12), $a_{2}$ must be known.

Proposition 3. Consider the system in (1) with the supertwisting control law (34)-(36) and the dual-layer adaptive scheme given by (9), (10) and (12). Then, for a sufficiently large $\lambda,(37)$ is forced to zero in finite time which guarantees the conditions for a 2-SM to exist are met. Furthermore the adaptive gains $k(t)$ in (9) and $r(t)$ in (12) remain bounded.

Proof: Consider $V(e, \delta)$ from (15) as a candidate Lyapunov function. Using arguments similar to those in the proof of Proposition 1

$$
\delta \dot{\delta} \leq-r_{0}|\delta|-r(t)|\delta|+|\delta| \frac{\bar{q} a_{2}}{\alpha}=\left(-r_{0}+e\right)|\delta|
$$

and it follows

$$
\dot{V} \leq\left(-r_{0}+e\right)|\delta|-\frac{1}{\gamma} e \dot{r}(t) \leq-r_{0} \sqrt{2} V^{1 / 2}
$$

This guarantees that in finite time $V=0$. This in turn guarantees finite time convergence of $\delta, e \rightarrow 0$ and the boundedness of $k(t)$ and $r(t)$ from (9) and (12).

Since $\delta=0$ in finite time, using (37), the following holds

$$
k(t)=\frac{1}{\alpha}\left|\bar{w}_{e q}(t)\right|+\varepsilon>\left|w_{e q}(t)\right|=|\varphi(t)|
$$

This means equation (33) holds, and provided $\lambda$ in (31) is sufficiently large, the 2-SM is maintained.

\subsection{A formulation when $a_{2}$ is unknown}

If $a_{2}$ is unknown the formulation (12) used in Section 4.1 is not applicable since $e(t)$ in (38) is unknown. Instead consider

$$
\dot{r}(t)= \begin{cases}\gamma|\delta(t)| & \text { if }|\delta(t)|>\delta_{0} \\ 0 & \text { otherwise }\end{cases}
$$

where $\gamma$ and $\delta_{0}$ are positive scalars. The stability of the dynamics $\delta(t)$ and $e(t)$ from (37) and (38) together with the adaptive laws (9) and (42) will now be investigated. (Note this formulation does not require knowledge of $a_{2}$.)

Proposition 4. Consider the system in (1) with the supertwisting control law (34)-(36) and the dual-layer adaptive scheme given by (9), (10) and (42). If $\delta_{0}$ satisfies

$$
\frac{1}{4} \epsilon^{2}>\delta_{0}^{2}+\frac{1}{\gamma}\left(\frac{\bar{q} a_{2}}{\alpha}\right)^{2}
$$

then the error $\delta(t)$ in (37) is forced to satisfy $|\delta(t)|<\epsilon / 2$ in finite time, and for a sufficiently large $\lambda$, the conditions for a 2-SM to exist are met. Furthermore the adaptive gains $k(t)$ in (9) and $r(t)$ in (42) remain bounded.

Proof: Using the Lyapunov function candidate $V(e, \delta)$ from (15), arguing as in the proof of Proposition 2, in finite time the inequality $|\delta(t)|<\epsilon / 2$ is guaranteed, and from (37) it follows

$$
k(t)>\frac{1}{\alpha}\left|\bar{w}_{e q}(t)\right|+\varepsilon / 2>\left|w_{e q}(t)\right|=|\varphi(t)|
$$

This means that equation (33) holds, and provided $\lambda$ from (31) is sufficiently large, a 2-SM is maintained. 


\section{Adaptive Continuous HOSMC}

Now consider the more generic case when

$$
\sigma^{(n)}(t)=u(t)+a(t)
$$

where the integer $n>2$, and $u(t)$ represents the control variable whilst $a(t)$ is an unknown disturbance. The objective is to force $\sigma, \dot{\sigma}, \ldots, \sigma^{(n)}=0$ in finite time. Consider a control law comprising two parts:

$$
u(t)=-u_{b}(t)-u_{s}(t)
$$

where

$$
u_{b}(\cdot)=\gamma_{1}|\sigma|^{\alpha_{1}} \operatorname{sign}(\sigma) \ldots+\gamma_{n}\left|\sigma^{(n-1)}\right|^{\alpha_{n}} \operatorname{sign}\left(\sigma^{(n-1)}\right)
$$

and

$$
u_{s}(t)=\lambda|s|^{1 / 2} \operatorname{sign}(s(t))+\int_{0}^{t} k(t) \operatorname{sign}(s(\tau)) d \tau
$$

where the auxiliary sliding variable

$$
s(t)=\sigma^{(n-1)}(t)+\int_{0}^{t} u_{b}(\tau) d \tau
$$

In (47) the scalars $\gamma_{1}, \gamma_{2}, \ldots, \gamma_{n}$ must be chosen such that the polynomial $p^{n}+\gamma_{n} p^{n-1}+\ldots+\gamma_{2} p+\gamma_{1}$ is Hurwitz and the scalars $\alpha_{1}, \alpha_{2}, \ldots, \alpha_{n}$ are chosen recursively as

$$
\alpha_{i-1}=\frac{\alpha_{i} \alpha_{i+1}}{2 \alpha_{i+1}-\alpha_{i}}, i=2, \ldots, n
$$

with $\alpha_{n+1}=1$ and $\alpha_{n}=\bar{\alpha}$. In (48) the positive scalar $\lambda$ must be chosen sufficiently large and the time varying gain $k(t)$ adapts according to the dual layer structure previously described in Section 4.

Theorem 1: Consider the system in (45) with uncertainty $a(t)$ which is twice differentiable and subject to $|\dot{a}(t)|<a_{1}$ and $|\ddot{a}(t)|<a_{2}$. Using the control law from (46) in (45) where $k(t)$ adapts according to the dual layer equations (9),(42) and assuming (43) is satisfied, then for sufficiently large $\lambda$, there exists an $\epsilon_{b} \in(0,1)$ such that for every $\bar{\alpha} \in\left(1-\epsilon_{b}, 1\right)$ the origin $\sigma, \dot{\sigma}, \ldots, \sigma^{(n)}=0$ is a finite time stable equilibrium point.

Proof: From equations (49) and (47)-(48), the dynamics associated with the auxiliary variable $s$ are described by

$$
\dot{s}(t)=a(t) \underbrace{-\lambda|s|^{1 / 2} \operatorname{sign}(s(t))-\int_{0}^{t} k(\tau) \operatorname{sign}(s(\tau)) d \tau}_{-u_{s}(t)}
$$

This is exactly equivalent to the set of equations in (34)-(36) and so arguing as in proof of Proposition 4, the dual layer adaptive structure induces a $2 \mathrm{SM}$ in finite time in which $\dot{s}=s=0$, and during the $2 \mathrm{SM}$ the control term $u_{s}$ exactly compensates for the uncertainty: i.e $a(t)=u_{s}(t)$. Therefore during a $2 \mathrm{SM}$

$$
\sigma^{(n)}=a(t)+u(t)=\underbrace{a(t)-u_{s}(t)}_{=0}-u_{b}(t)=-u_{b}(t)
$$

and therefore from the definition of $u_{b}$ in (47), the sliding motion is governed by

$$
\sigma^{(n)}=-\gamma_{1}|\sigma|^{\alpha_{1}} \operatorname{sign}(\sigma) \ldots-\gamma_{n}\left|\sigma^{(n-1)}\right|^{\alpha_{n}} \operatorname{sign}\left(\sigma^{(n-1)}\right)
$$

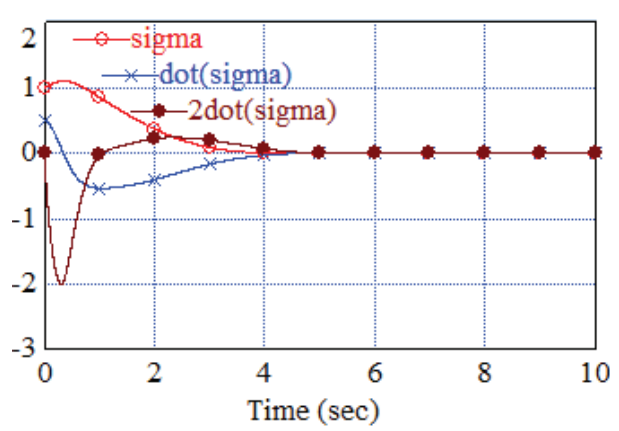

Figure 7: Evolutions of $\sigma(t), \dot{\sigma}(t)$ and $\ddot{\sigma}(t)$

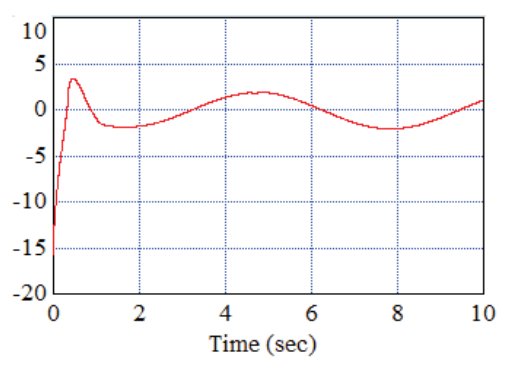

Figure 8: Evolutions of $u(t)$

From Proposition 8.1 presented in Bhat \& Bernstein (2005), there exists an $\epsilon_{b} \in(0,1)$ such that for every $\bar{\alpha} \in\left(1-\epsilon_{b}, 1\right)$ the origin $\sigma, \dot{\sigma} \ldots, \sigma^{(n)}=0$ is a finite time stable equilibrium $\quad \square$

Remark 6: The continuous controller (46) can be claimed to be a continuous HOSM controller for the system in (1), since it drives $\sigma, \dot{\sigma}, \ldots, \sigma^{(n)} \rightarrow 0$ in finite time in the presence of the smooth disturbance $a(t)$ with bounded derivatives $|\dot{a}(t)| \leq a_{1}$ and $|\ddot{a}|<a_{2}$.

\subsection{An Example}

Consider the system in (45) with relative degree $n=3$. The coefficients of the underlying Hurwitz polynomial $p^{3}+\gamma_{3} p^{2}+$ $\gamma_{2} p+\gamma_{1}$ associated with the controller are selected as $\gamma_{1}=$ $8, \gamma_{2}=12, \gamma_{3}=6$. The exponents $\alpha_{1}, \alpha_{2}, \alpha_{3}$ in equation (47) are calculated based on the seed $\bar{\alpha}=0.8$ in equation(50). Explicitly they are given by $\alpha_{1}=0.56, \alpha_{2}=0.66$ and $\alpha_{3}=0.8$. The initial conditions are selected as $\sigma(0)=1, \dot{\sigma}(0)=0.5$, $\ddot{\sigma}(0)=0$. The disturbance in (45) is taken as $a(t)=2 \sin (t)$. The parameter $\lambda=4.75$ and the other parameters that are used in simulations are $r_{0}=1, \gamma=800$ and $\alpha=0.99$; and $\delta_{0}=0.01$ and $\epsilon=0.15$. The time constant of the filter is $\tau=0.01$, starting with zero initial conditions. It is assumed that $a_{2}$ exists but is unknown. In this case the adaptive control HOSMC in (46)-(49) is employed. The results are shown in Figures 7-10. Figure 7 shows the convergence of $\sigma, \dot{\sigma}$ and $\ddot{\sigma}$ to zero in finite time despite the presence of the disturbance, using a smooth control action (Figure 8). Figure 9 shows that $\rho(t)$ does not track the unknown bound $a_{2}$, but nevertheless $k(t)$ closely tracks $|a(t)|$ (Figure 10). 


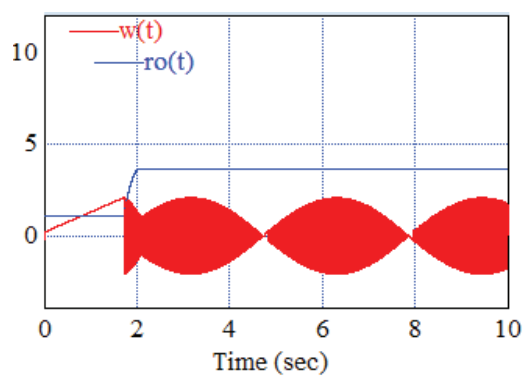

Figure 9: Evolutions of $w(t)$ compared to $\rho(t)$

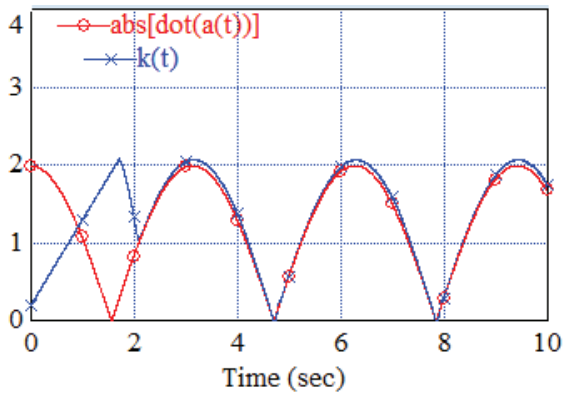

Figure 10: Evolutions of $k(t)$ compared to $|a(t)|$

\section{Conclusion}

This paper has proposed a new adaptive scheme for conventional, super-twisting and higher-order sliding mode control algorithms. It is based on a novel dual layer nested approach and relies on the availability of an online approximation of the equivalent control associated with the (possibly embedded) switched terms. The assumptions on the uncertainty and the required knowledge about the bounds on the magnitude and derivative of the uncertainty are weaker than those in the comparable literature. In particular in its most general form, the scheme does not require information about the upper-bound of the disturbance and its derivative. Here the adaptive ideas are deployed for conventional, super-twisting and higher-order control structures but the underlying ideas can be applied to other sliding mode control structures. The schemes are robust to imperfections in the estimates of the equivalent control by choice of two specific scalar parameters. These trade-off robustness, against the degree of conservatism over-bounding the uncertainty. The simulations presented in the paper confirm the effectiveness and simplicity of the approach.

\section{Acknowledgements}

We would like to acknowledge one of the anonymous reviewers for the helpful technical suggestions during the review cycle, which has helped improve the content of the paper.

\section{References}

H. Alwi and C. Edwards. An adaptive sliding mode differentiator for actuator oscillatory failure case reconstruction. Automatica, 49:642-651, 2013.
G. Bartolini, A. Levant, F. Plestan, M. Taleb, and E. Punta. Adaptation of sliding modes. IMA Journal of Mathematical Control and Information, 30:885300, 2013.

S. Bhat and D.S. Bernstein. Geometric homogeneity with applications to finitetime stability. Math. Control Signals Systems, 17:101-127, 2005.

C. Edwards and S. K. Spurgeon. Sliding Mode Control: Theory and Applications. Taylor \& Francis, 1998.

A.F. Filippov. Differential equations with discontinuous right hand-sides. American Mathematical Society Translations, 42:199-231, 1964.

Y.-J. Huang, T.-C. Kuo, and S.-H. Chang. Adaptive sliding-mode control for nonlinear systems with uncertain parameters. IEEE Transactions on Systems, Man, and Cybernetics, Part B: Cybernetics, 38:534 - 539, 2008.

H.K. Khalil. Nonlinear Systems. Prentice Hall, Englewood Cliffs NJ., 1992.

H. Lee and V.I. Utkin. Chattering supression methods in sliding mode control systems. Annual Reviews in Control, 31:179-188, 2007.

A. Levant. Higher order sliding modes, differentiation and output feedback control. Interantional Journal of Control, 76:924-941, 2003.

A. Levant. Quasi-continuous higher order sliding mode controllers. IEEE Transactions on Automatic Control, 50:1812-1816, 2006.

J. A. Moreno and M. Osorio. A Lyapunov approach to second-order sliding mode controllers and observers. In 47th IEEE Conference on Decision and Control, pages 2856-2861, 2008.

F. Plestan, Y. Shtessel, V. Bregeault, and A. Poznyak. New methodologies for adaptive sliding mode control. International Journal of Control, 83:19071919, 2010.

Y. Shtessel, C. Edwards, L. Fridman, and A. Levant. Sliding Mode Control and Observation. Birkhauser, 2013.

Y. Shtessel, M. Taleb, and F. Plestan. A novel adaptive-gain supertwisting sliding mode controller: Methodology and application. Automatica, 48:759769, 2012 .

M. Taleb, A. Levant, and F. Plestan. Electropneumatic actuator control: solutions based on adaptive twisting algorithm and experimentation. Control Engineering Practice, 21:727-736, 2013.

V. I. Utkin. Sliding Modes in Control Optimization. Springer-Verlag, Berlin, 1992.

V.I. Utkin and A.S. Poznyak. Adaptive sliding mode control. In B. Bandyopadhyay, S. Janardhanan, and S.K. Spurgeon, editors, Advances in Sliding Mode Control: Concepts, Theory and Implementation, volume 440 of LNCIS, pages 21-53. Springer Berlin, 2013.

V.I. Utkin and A.S. Poznyak. Adaptive sliding mode control with application to super-twisting algorithm: Equivalent control method. Automatica, 49:3947, 2013. 